Retos, I0(19), 2020

\title{
Identificación de la percepción del turista hacia el destino Cuenca-Ecuador, mediante la "Netnografía"
}

\section{Identification of the tourist's perception towards the destination Cuenca-Ecuador, through "Netnography"}

\begin{abstract}
Nicolás E. Morales Vásquez es Ingeniero Comercial por la Universidad Politécnica Salesiana (Ecuador) (nicolasmv@outlook. com) (https://orcid.org/0000-0003-3214-4212)
\end{abstract}

Dra. Gliceria P. Gómez Ceballos es profesora e investigadora en el Área de Administración y Economía de la Universidad Politécnica Salesiana (Ecuador) (ggomez@ups.edu.ec) (https://orcid.org/0000-0001-6955-3293)

\begin{abstract}
Resumen
Esta investigación analiza la necesidad y conveniencia de demostrar que la Netnografía es un método que aporta al desarrollo del turismo, ya que contribuye a determinar los gustos y preferencias con relación a los recursos/atractivos existentes en la localidad y el nivel de satisfacción de las necesidades en los consumidores. El objetivo general es identificar percepciones de los turistas para el destino Cuenca-Ecuador y la metodología seleccionada para el estudio, fue la propuesta por Vargas (2011), quien establece como pasos del procedimiento: la definición del tópico a investigar, las comunidades virtuales donde se extraerán los datos y por último, la interpretación de la información. El universo de la investigación integró la totalidad de comentarios extraídos de la página de Facebook de Visit Cuenca Ecuador y el foro de turismo de la ciudad de Cuenca en Trip Advisor. El análisis arrojó como resultado la percepción de los turistas con relación a los atributos que oferta la ciudad, la existencia de una mayor afluencia de turismo nacional y segmentación de turistas extranjeros por su procedencia, destacándose América Latina y Caribe; se observa que el atractivo más importante en orden de preferencia es el área arquitectónica y los atractivos naturales del entorno; hallazgos que podrán ser tomados en cuenta para el diseño de futuras estrategias de comercialización del destino.
\end{abstract}

\begin{abstract}
This investigation analyzes the necessity and convenience of demonstrating that the "Netnography" is a method that contributes to the development of tourism, because it contributes to determine the tastes and preferences in relation to the resources/attractions existing in the locality and the level of satisfaction of the consumer's needs, the objective general is to identify perceptions of tourists for the destiny Cuenca Ecuador, the methodology selected for the study was the proposed by Vargas (2011), who stablishes as steps of the procedure: the definition of the topic to investing, the virtual communities where the data will extracted and finally, the interpretation of the information. The universe of the research integrated totality of comments extracted from the Facebook's page Visit Cuenca and the tourist forum of the Cuenca's city in Trip Advisor. The analysis show as results the perception of the tourists in relation to the attributes offered by the city, the existence of a greater influx of Ecuadorian tourism into the city and the composition by origin foreign segments, including tourists from Latin América and Caribbean, it's observed that the most important attraction in order of preference was its architectural area and the natural attractions of the environment; findings that can be taken into account for the design of future marketing strategies for the destination.
\end{abstract}

\section{Palabras clave I keywords}

Atributos, perfil turista, destino turístico, mercadeo, minería de datos.

Attributes, tourist profile, tourist destination, marketing, data mining.

Cómo citar: Morales Vásquez, N. E., y Gómez Ceballos, G. P. (2020). Identificación de la percepción del turista hacia el destino Cuenca-Ecuador, mediante la "Netnografía". Retos Revista de Ciencias de la Administración y Economía, 10(19), 81-97. https://doi.org/10.17163/ret. n19.2020.05 


\section{Introducción}

Ecuador en las últimas décadas ha reflejado un incremento del turismo, desarrollando un nuevo potencial para diversificar la económica del país. Para ello, se deben aprovechar las experiencias de otros países que permita desarrollar un modelo turístico sostenible (Castillo, Roget \& Rozas, 2015). El país cuenta con una estructura productiva con mucha dependencia del sector primario, por lo tanto, existe la necesidad de diversificar la matriz productiva y la búsqueda de alternativas de ingreso que mejoren su economía —cuestión que se expresa claramente como voluntad política en (SENPLADES, 2012).

Para alinearse con este propósito, resulta necesario incorporar nuevas tecnologías al proceso de gestión turística, dado que hoy día desde el enfoque de demanda, los turistas básicamente organizan su viaje a través del uso de dispositivos tecnológicos; de manera que para dar respuesta a esta tendencia, es importante que desde la oferta, exista una respuesta coherente con estas expectativas. Solo con relación al mercado interno, los datos del INEC (2017) arrojan que el uso de smartphones o teléfonos inteligentes ha aumentado en el transcurso del tiempo, indicando que en el año 2017 un 63.4\% de las personas poseen un teléfono inteligente en comparación con 2016 que constaba con un 52.9\%, lo que representa un aumento del 10.7\%.

Con relación al motivo del uso del internet, en el 2017 el 40.7\% de la población utiliza para obtener información, comunicación general (31\%), educación y aprendizaje $(21.10 \%)$, razones de trabajo $(3,3 \%)$ y para otras actividades con el $3.9 \%$. El uso de las TIC juega un papel importante para el crecimiento empresarial, toma de decisiones y la creación de nuevos modelos de negocios diversificando la interacción entre los turistas y las agencias de viaje tomando como referencia una nueva estructura tecnológica (Tafur Avilés et al., 2018).

Como parte del impulso a la política de desarrollo del turismo, el Ministerio de Turismo del Ecuador (MINTUR), en el año 2017 instauró una alianza con Amadeus IT Group con la finalidad de promover a Ecuador como un destino turístico preferido por los extranjeros, mediante el uso de una plataforma llamada "AMADEUS", que accederá a investigar y analizar las tendencias actuales —en base a cuyos resultados se formularon estrategias para la promoción del país- aplicándolo de forma eficiente esas estrategias a diversos mercados (MINTUR, 2017). Estas son señales positivas que desde las políticas gubernamentales denotan el interés y preocupación en el país por la incorporación de los avances tecnológicos al proceso de gestión del turismo.

En el ámbito de las microempresas de la ciudad de Cuenca, Aucay Piedra y Herrera Torres (2017) manifiestan que el 97\% de los establecimientos hace uso de las redes sociales diariamente para actividades de comunicación, ya que revelan ventajas obtenidas por la administración de redes sociales y aplicaciones móviles para la comunicación y mercadotécnica. Para aprovechar al máximo las oportunidades de negocio que brindan los servicios móviles vinculados al turismo, es necesario realizar una segmentación bien detallada del tipo de visitante que ingresa al país, existiendo varios nichos con alto grado de potencialidad para el aumento de ingresos al segmento turístico (Quezada-Sarmiento et al., 2018).

La revisión bibliográfica realizada con el objetivo de identificar el estado del arte acerca de los elementos que sustentan el análisis de esta investigación, mues- 
tra con relación a la importancia del uso de las redes sociales en los procesos de gestión que, Dell Innocenti (2012), lo resume de esta manera: acortan la relación empresa-cliente incrementando su dinámica, estrechan la vinculación online-offline, permiten la integración de herramientas como medios de publicidad y por último destaca, el valor de aplicar medios digitales y no digitales aprovechando las bondades que cada uno provee; aportan además a la atención de necesidades a nivel individual de sus clientes. Sakulsureeyadej (2011) agrega que, la mayoría de viajeros tienen altas expectativas en recibir productos o servicios personalizados para satisfacer sus exigencias y las empresas por tanto, deben disponer de herramientas para recolectar y controlar la información.

Desde otra perspectiva que sostiene los planteamientos anteriores, es necesario tener en cuenta la necesidad de promover el uso de las aplicaciones móviles y de los teléfonos inteligentes (smartphones) a nivel global, éstos son los que abren la posibilidad de utilizar estas aplicaciones, cuyo crecimiento es exponencial y su uso difiere en dependencia del segmento de mercado por edades, destacando que el grupo etario comprendido entre los 35 y 55 años es el que mayor dinámica de crecimiento ha tenido en los últimos 3 años (Saura, Sánchez, \& Reyes, 2017).

Estos autores, además, enfatizan en el orden de utilización de estas tecnologías en los últimos tres años. Las aplicaciones móviles de comunicación y redes sociales las más utilizadas con un 47\%, seguidas de aplicaciones de negocios, finanzas, compras y deportes (Saura et al., 2017); el teléfono inteligente se convierte en un excelente intermediario entre el viajero y el destino, ya que le permite interactuar directamente con los agentes para decidir alternativas de uso de su tiempo de ocio (Quijano, Arellano, \& Naranjo, 2017). Por otro lado existe un incremento en el uso de teléfonos móviles para obtener información, utilizada por 96 de cada 100 habitantes en el mundo, siendo visto como un medio para ofrecer servicios a través de internet (Desplas \& Mao, 2014; Dina \& Sabou, 2012; Kang \& Schuett, 2013). Los autores citados hacen referencia a que los turistas prefieren utilizar redes sociales como medio de comunicación on-line para la toma de decisiones y organización de su viaje al momento de elegir su destino turístico.

Los autores Marín y Torres (2017) expresan que, la banca ha estado experimentando con el uso de elementos de juego en las aplicaciones móviles con la finalidad de incrementar la fidelidad de los clientes, sin embargo, no se menciona a la Netnografía como medio para la obtención de información de sus clientes (gustos, preferencias, necesidades, sugerencias) para desarrollar o mejorar la fidelización de sus clientes.

Quijano et al. (2017) destacan que las estadísticas demuestran que el uso de dispositivos móviles en el ámbito del turismo se ha generalizado; con un alto porcentaje se identifica a los turistas provenientes de EEUU (85\%), seguido de europeos con un $50 \%$ en diferentes modalidades de uso; lo cual tiene especial significación para Ecuador, dado que el mayor emisor de turismo extranjero actual se encuentra en EEUU y a futuro, se debe volver la vista al mercado europeo, dadas las potencialidades existentes en el país con relación a la presencia de recursos/atractivos naturales y su preferencia por parte de los turistas que proceden de estas latitudes.

La dinámica de uso de estas tecnologías ha puesto retos a la investigación de mercados; este paso es el punto de partida de cualquier proceso de gestión, ya que 
permite analizar las variables del entorno con respecto a la competencia, el macro y micro entorno y especialmente la demanda - aspectos que condicionan la oferta turística. El uso de las redes sociales como fuente de información de destinos turísticos ha crecido dejando a un lado la forma tradicional de acceder a los viajes para el disfrute del tiempo de ocio. Por ello surge la Netnografía como herramienta tecnológica que accede a indagar el comportamiento de los usuarios en páginas webs, mediante un análisis estadístico de los datos obtenidos. Gebera (2008) describe a la Netnografía como un método para aprovechar al máximo la información que proporciona la red; establece como base a la etnografía para comprobar el comportamiento del consumidor en los diferentes espacios virtuales.

El uso de la Netnografía ha permitido establecer un conjunto de varios factores que servirán de base para su aplicación a futuro en varias empresas especializadas en su sector, ya que facilita el conocimiento amplio de los consumidores finales que forman parte de los sitios virtuales, integrándoles como un objetivo estratégico para la promoción y distribución (Colordo \& Prutsky, 2004).

Esta técnica logra obtener una amplia diferencia en comparación con la etnografía en cuanto a la generación de mayor conocimiento fiable, evita que el investigador se involucre directamente en el campo de investigación, impide cualquier tipo de manipulación de la información, más sencilla y de rápida aplicación, acceso inmediato a la información (Romeo, Abad, Forgas \& Huertas, 2014).

En el ámbito internacional, los países tienen un mayor interés en manejar las redes sociales como medio de promoción turística, destacándose Instagram como la red con más interacción entre sus usuarios, siendo idóneo para la comunicación turística (Gutiérrez, Sánchez \& Galiano, 2018). Mientras que (Celdrán, Mazón \& Giner, 2018) hacen referencia a que, en la actualidad, obtener información turística a través del uso de aplicaciones móviles, es fundamental para promover el disfrute de una mejor experiencia de parte del turista y con ello, incrementar los niveles de competitividad, cuestión que ocupa hoy el centro de atención en los países que gestionan el turismo como una alternativa de desarrollo.

Desde esta perspectiva, las empresas turísticas ecuatorianas deben utilizar las redes sociales para proporcionar la mayor cantidad de información con relación a su propuesta turística, así como enfocarse en aprovechar la información extraída de los comentarios de sus seguidores, con el propósito de diseñar nuevas ofertas y estrategias de posicionamiento con base en las preferencias de los turistas, aun cuando a diferencia de la forma tradicional de comunicación y manejo de información, la extensa cantidad de datos cualitativos que brindan las redes sociales obligan a buscar alternativas para su medición (Fisher, 2009); definir cuáles son los puntos claves para conseguir diferenciación con respecto a su competencia, debido a que los turistas revisan primero blogs, foros de turismo, para conocer sobre su lugar de destino (Montserrat, 2014); entender que el uso de redes sociales es esencial para la atracción de clientes y el mantenimiento de su fidelidad (Morales, 2016); tomar en consideración que las redes sociales incluso, se han vuelto más impulsivas en la decisión de visitar nuevos destinos turísticos (Sotiriadis \& Van Zyl, 2013).

En consecuencia, las aplicaciones móviles para viajes y turismo no solo están aumentando exponencialmente, sino que están cambiando la forma en que el turista 
busca información, se comporta, consume y toma decisiones; además los teléfonos inteligentes y las aplicaciones móviles hacen que el viajero pueda alinear sus necesidades, preferencias, recursos y restricciones de tiempo, con la oferta de servicios y actividades en el lugar de destino, lo que le brinda más libertad que nunca a la hora de movilizarse.

Existen, por tanto, claras certidumbres acerca de la importancia que en la actualidad tiene el uso de aplicaciones móviles para la orientación del turista y el manejo de información, que permita estructurar una oferta coherente con sus expectativas. Sin embargo, no se pudo evidenciar ninguna experiencia documentada vinculada al uso de la Netnografía como herramienta de investigación de mercados; por ello se precisó como objetivo general de este trabajo investigativo, identificar la percepción de los turistas para el destino Cuenca mediante la aplicación metodológica denominada "Netnografía", la misma que es idónea para la investigación, ya que nos brinda la facilidad de obtener y analizar diversos comentarios, palabras, que los turistas realizan en las diversas comunidades virtuales relacionadas al turismo de Cuenca.

\section{Materiales y métodos}

Para el desarrollo de la investigación se utilizó la minería de datos, que facilita el descubrimiento de relaciones, patrones y tendencias, examinando grandes cantidades de datos extraídos de distintas bases de datos, la web, enlazados con programas estadísticos para analizar de forma cuantitativa la información obtenida, como, por ejemplo, número de comentarios positivos y negativos, particularmente se aplicó el método de Netnografía, a partir del procedimiento propuesto por Vargas (2011). Los pasos a seguir según el método seleccionado son:

\subsection{Definición pregunta de investigación o tópico a investigar}

La pregunta de investigación es: identificar factores en las diferentes comunidades virtuales relacionadas al turismo que aporten información para determinar la percepción del turista con destino turístico a la ciudad de Cuenca.

Las unidades de análisis seleccionadas fueron: Redes Sociales: Página de Facebook Visit Cuenca Ecuador; Foros de turismo: Foro de Trip Advisor Cuenca Ecuador.

Se identificaron las siguientes variables relacionadas con las preferencias del turista: Atractivos turísticos (culturales, naturales), calidad de servicios de alojamiento y restauración, percepción de precios del destino, seguridad, hospitalidad, clima, así como también su lugar de origen.

\subsection{Identificación y selección de comunidad}

Para seleccionar las comunidades se siguen los criterios de relevancia, actividad, interactividad, sustancialidad, heterogeneidad y riqueza de datos.

Cabe recalcar que se realizó una observación a las diferentes páginas web de agencias de viaje que brindan un paquete turístico hacia la ciudad de Cuenca, valorando su calidad en atención al contenido de la oferta y especialmente se tomó en cuenta la interactividad permitida entre la página y sus usuarios. Con base en los criterios mencionados anteriormente se ha seleccionado las siguientes comunidades: 
- Página de Facebook Visit Cuenca Ecuador: Esta página permite acceder a los comentarios y opiniones de parte de los turistas que han visitado la ciudad y obtener información acerca de las personas que tienen previsto visitarla en el futuro; este objetivo se evaluó mediante el análisis del contenido de las preguntas realizadas en el foro vinculadas a las preferencias del turista. La selección obedece a que es la única que posee un espacio de interactividad.

- Trip Advisor Foro Cuenca Ecuador: Este foro goza de popularidad entre los viajeros, ya que proporciona información sobre el destino, hoteles, lugares turísticos, restaurantes entre otros, aportó comentarios u opiniones de las turistas con relación a temas como seguridad, clima, transporte, lugares a visitar.

\section{Recolección de datos}

El procedimiento para la recolección de datos se realizó de forma automática y manual, alineados con el procedimiento seleccionado, para ello se utilizaron dos softwares:

Facepager y Netvizz: Estos softwares permiten estructurar una base de datos con los comentarios y publicaciones (posts) realizados en páginas o grupos públicos en Facebook, en este caso, se utilizó para extraer información de la página de Visit Cuenca Ecuador en Facebook; de ella se obtuvieron comentarios, tanto de las publicaciones realizadas por la página y de la sección “Opiniones” de la misma.

Estos softwares presentan limitaciones, ya que solamente se puede obtener información de los usuarios que comparten o comentan ésta públicamente. Valoradas las bondades de ambos softwares se decidió utilizar solamente el Facepager, puesto que se verificó que Netvizz da resultados similares; para el procesamiento estadístico de los datos, se empleó el software estadístico SPSS IBM Estatistics 22.

El software Facepager, permite establecer un rango de fechas para la extracción de datos, se decidió extraer los comentarios realizados en la página de Facebook de Visit Cuenca Ecuador entre las fechas de enero 2017 hasta febrero del 2019.

Para el procedimiento manual, se creó una base de datos con todos los comentarios realizados por los viajeros en el foro de Cuenca-Ecuador en la página web de Trip Advisor; posteriormente se analizó cada comentario con el objetivo de eliminar publicaciones erróneas o que no tienen concordancia con el objeto de estudio. A continuación, se utilizó el programa AntCont que facilitó la elaboración de un listado con las palabras más repetidas de la base de datos obtenida mediante el software Facepager.

\section{Análisis de datos e interpretación y presentación de resultados}

Realizado el levantamiento de los datos, se procedió a revisar cada uno de los mensajes y se clasificaron en correspondencia con las variables identificadas en el estudio, posteriormente se procesó la información a través del software SPSS versión 22.

\section{Resultados}

La presente sección consta de dos partes, en la primera se muestran los resultados sobre la adecuación con los propósitos de la investigación de las agencias nacionales y 
extranjeras que operan en el destino; en la segunda se exponen los resultados obtenidos a través del uso de los softwares Facepager, IBM Estatistics y el programa AntCont.

\subsection{Primera Parte}

Se seleccionaron 13 agencias nacionales e internacionales (véase Tabla 1), que gestionan tours o paquetes turísticos hacia el destino Cuenca-Ecuador, los criterios de búsqueda de información fueron:

1. Si la página web consta con un espacio para que los turistas den sus opiniones o comentarios sobre sus experiencias cuando disfrutan del tour.

2. Se identificó el número de comentarios de las páginas que brindan esa opción.

\section{Tabla 1. Listado de agencias nacionales y extranjeras que ofertan tours hacia el destino Cuenca-Ecuador}

\begin{tabular}{|l|l|l|l|}
\hline \multicolumn{3}{|c|}{ Nacionales } & \multicolumn{2}{c|}{ Internacionales } \\
\hline 1 & Sol Caribe Travel Agency & 14 & Get Your Guide \\
\hline 2 & Spring Travel Tour Operator & 15 & Expedia \\
\hline 3 & Nova Travel EC & 16 & Atrápalo \\
\hline 4 & Corpo Viajes Agency Travel & 17 & Mundo Travel \\
\hline 5 & Virgen Rent a Car & 18 & Trip \\
\hline 6 & La moneda Viaje Turismo \& Eventos & 19 & Visit a City \\
\hline 7 & Vive Now & 20 & Tours 4 Fun \\
\hline 8 & Cazhuma Tours Travel Agency & 21 & Anywhere \\
\hline 9 & Terra Diversa Ecuador Explorer & 22 & Gray Line \\
\hline 10 & Cite Tour & 23 & Price Travel \\
\hline 11 & Expediciones Apullacta & 24 & Viator a Trip Advisor Company \\
\hline 12 & Tours Costa Ecuador & 25 & Inspirock \\
\hline 13 & Turi Bus & 26 & Happy Gringo \\
\hline
\end{tabular}

Fuente: Elaboración propia del autor

Los resultados arrojan, que las agencias nacionales no cuentan con ningún espacio de comentarios u opiniones y 4 de 13 agencias internacionales sí lo poseen, lo que representa un 15\%, tal como se muestra en el gráfico 1 . 


\section{Gráfico 1. Porcentaje de agencias nacionales e internacionales con un espacio para comentarios}

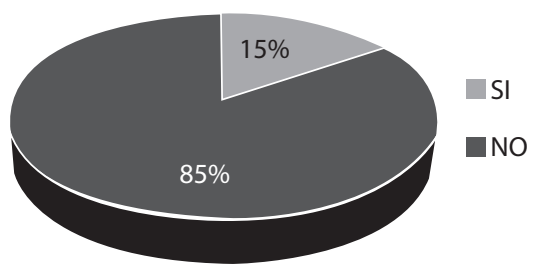

Fuente: Elaboración propia.

Ello limita el estudio a 2 fuentes (Trip Advisor y Visit Cuenca), por lo cual se ha excluido al resto de las agencias por no disponibilidad de información.

La observación realizada a las páginas web, arroja que éstas solo ofrecen información básica sobre la oferta (Tabla 2), a pesar de contar con un espacio para comentarios no se aprovecha adecuadamente esta disponibilidad.

Tabla 2. Número de comentarios en las websites de las agencias de viaje nacional e internacional

\begin{tabular}{|l|l|c|c|c|c|c|}
\hline No. & \multicolumn{1}{|c|}{ Agencia de Viaje } & $\begin{array}{c}\text { Cantidad de } \\
\text { comentarios u } \\
\text { opiniones }\end{array}$ & No. & \multicolumn{1}{|c|}{$\begin{array}{c}\text { Agencia } \\
\text { de Viaje }\end{array}$} & $\begin{array}{c}\text { Cantidad de } \\
\text { comentarios } \\
\text { u opiniones }\end{array}$ \\
\hline \multicolumn{1}{|c|}{ Nacionales } & - & 14 & Get Your Guide & 1 \\
\hline 2 & Sol Caribe Travel Agency & - & 15 & Expedia & - \\
\hline 3 & Spring Travel Tour Operator & Nova Travel EC & - & 16 & Atrápalo & - \\
\hline 4 & Corpo Viajes Agency Travel & - & 18 & Trip & - \\
\hline 5 & Virgen Rent a Car & - & 19 & Visit a City & - \\
\hline 6 & $\begin{array}{l}\text { La Moneda Viaje Turismo \& } \\
\text { Eventos }\end{array}$ & - & 20 & Tours 4 Fun & 0 \\
\hline 7 & Vive Now & - & 21 & Anywhere & - \\
\hline 8 & $\begin{array}{l}\text { Cazhuma Tours Travel Agency } \\
\text { \& Tour }\end{array}$ & - & 22 & Gray Line & - \\
\hline 9 & TerraDiversa Ecuador Explorer & - & 23 & Price Travel & 0 \\
\hline 10 & Cite Tour & - & 24 & $\begin{array}{l}\text { Viator A Trip } \\
\text { Advisor Company }\end{array}$ & 1 \\
\hline 11 & Expediciones Apullacta & - & 25 & Inspirock & - \\
\hline 12 & Tours Costa Ecuador & - & 26 & Happy Gringo & - \\
\hline 13 & Turi Bus & - &
\end{tabular}

Nota: El símbolo de guion (-) significa que la página no consta de un espacio para realizar un comentario. Fuente: Elaboración propia del autor. 
Se corroboran las evidencias encontradas en estudios anteriores Vázquez (2018), donde se comparan resultados de 20 empresas de turismo en base a la variable calidad de sus páginas web, y el factor interactividad se mantiene en niveles muy bajos (Gráfico 2).

\section{Gráfico 2. Valoración de calidad de las páginas webs de intermediación turística}

5

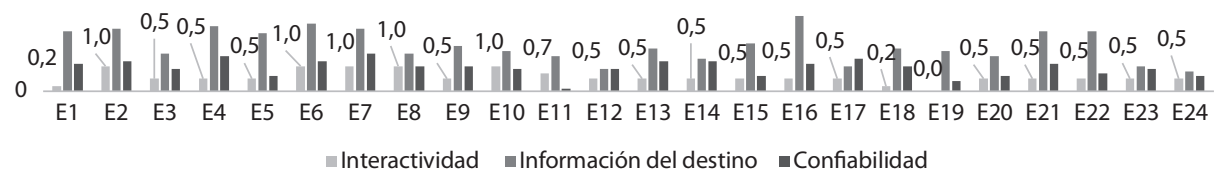

Nota: Abreviatura E1 significa Empresa 1. Fuente: Vázquez, J. P. (2018). Obtenido de https://bit.ly/2KlZrjx

\subsection{Segunda Parte}

Recolección de información de forma manual y automática.

Se listó el total de los comentarios de la página de Trip Advisor en la sección de foros vinculados a la ciudad de Cuenca; fueron válidos 51, para lo cual no se utilizó un software, debido a que se realizó de forma manual la obtención de información.

Posteriormente, se realizó la recolección de información de forma automática; para ello se utilizó la herramienta tecnológica Facepager, obteniendo información entre las fechas del 1 de enero del 2017 hasta el 1 de febrero del año 2019, de la página de Facebook Visit Cuenca, que sumados hacen un total de 249 comentarios recolectados, el total entre ambas plataformas (Foro Trip Advisor y Visit Cuenca) fue de 300 comentarios.

\section{Gráfico 3. Cantidad de comentarios recolectados de forma manual y automática}

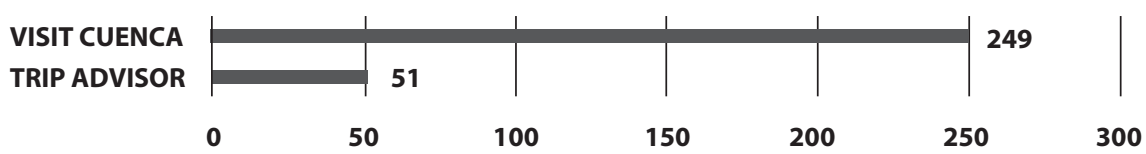

Fuente: Elaboración propia.

Se procedió a realizar un análisis de los comentarios mediante el software AntCont, para determinar la frecuencia de las palabras o atributos que más se repiten en los comentarios de los usuarios, tanto del foro de Trip Advisor como de la página de Facebook de Visit Cuenca.

Se calculó el índice de frecuencia, con relación a las palabras que reflejen la percepción de los turistas con respecto a la ciudad de Cuenca, haciendo énfasis en Atractivos turísticos (culturales, naturales), calidad de servicios de alojamiento y 
restaurantes, percepción de precios del destino, seguridad, hospitalidad, clima, y se ha escogido palabras que se repitan más de 4 veces, según se ilustra en el gráfico 4 , lo que da como resultado que la percepción de precios no está jerarquizada como atributo de preferencia.

\section{Gráfico 4. Frecuencia de palabras más utilizadas por los turistas}

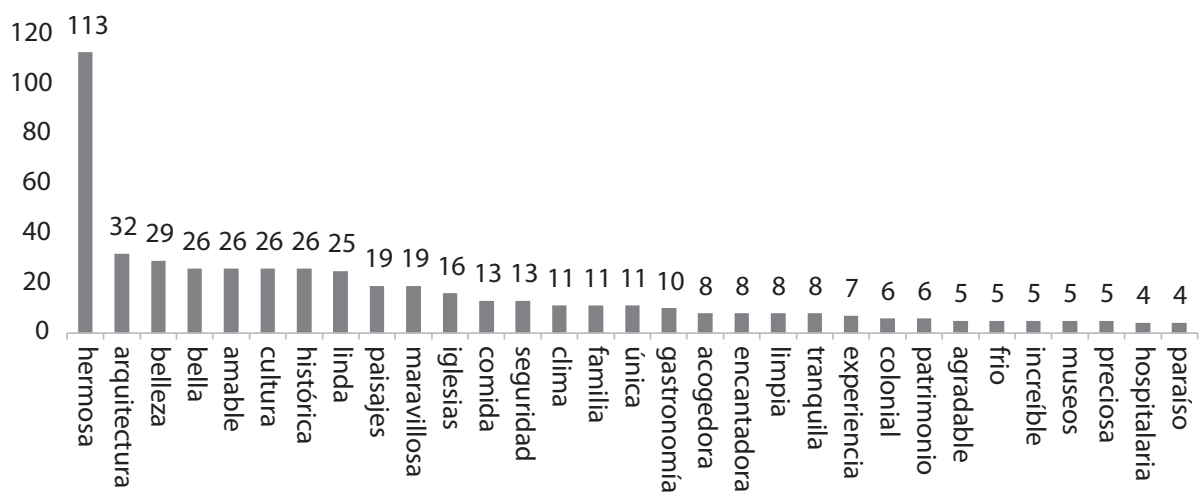

Fuente: Elaboración propia

Estos resultados (comentarios), se procesaron para un análisis estadístico mediante el software SPSS IBM Estatistics, aplicando la opción de tablas cruzadas entre las variables, País y Género, dando como resultado que los países de mayores niveles de emisión son Colombia, Perú, Argentina, seguido por Estados Unidos y España - como se observa en la Tabla 3- ello reafirma los resultados del Grupo de investigación en Economía Regional (GIER) que en el año 2018, asegura que los turistas que más visitan la ciudad de Cuenca proceden de América Latina (35.3\%).

Tabla 3. Personas con más participación en base a su país de origen

\begin{tabular}{|l|c|c|c|}
\hline \multirow{2}{*}{ País } & \multicolumn{2}{c|}{ Género } & \multirow{2}{*}{ Total } \\
\cline { 2 - 4 } & Femenino & Masculino & \\
\hline Ecuador & 114 & 62 & 176 \\
\hline EEUU & 30 & 23 & 53 \\
\hline Perú & 14 & 25 & 39 \\
\hline Colombia & 9 & 8 & 17 \\
\hline Argentina & 5 & 8 & 13 \\
\hline España & 7 & 0 & 7 \\
\hline
\end{tabular}

Nota: Basado en los resultados dados por el software SPSS IBM Estatistics.

Fuente: Elaboración propia 
Las preferencias de los turistas al seleccionar la ciudad de Cuenca como destino turístico se sitúan en torno a variables como "ciudad hermosa, arquitectura, gente amable, gastronomía”, (fueron las más comentadas, véase gráfico 5) y los factores como seguridad, clima, cultura y naturaleza fueron menos (gráfico 6).

\section{Gráfico 5. Factores que los turistas perciben de la ciudad de Cuenca}

Ciudad hermosa

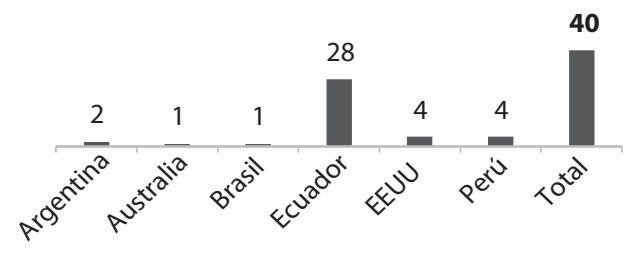

Gente amable

24

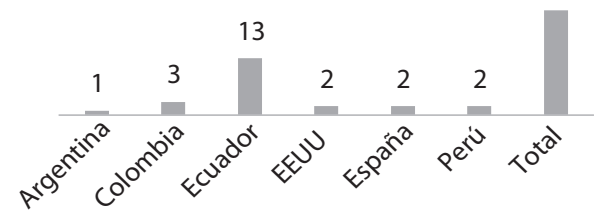

Arquitectura

26

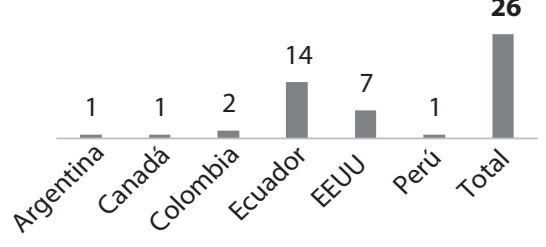

Gastronomía

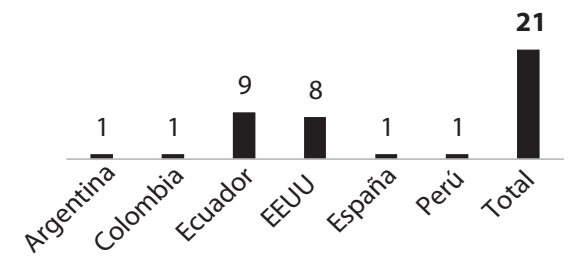

Fuente: Elaboración propia.

Gráfico 6. Factores que los turistas perciben de la ciudad de Cuenca

Cultura

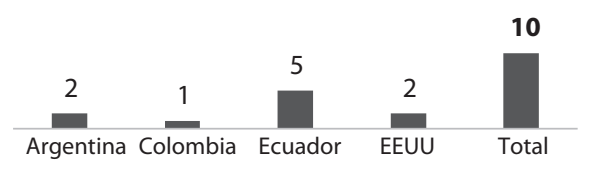

Segura

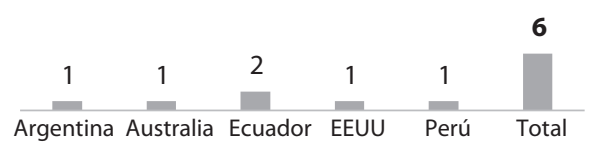

Naturaleza

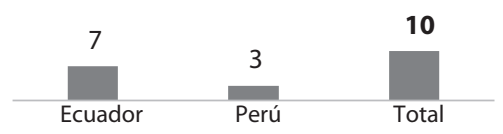

Clima agradable

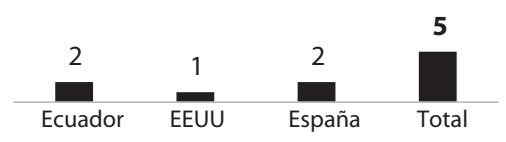

Fuente: Elaboración propia. 
Se observa, además, una mayor participación de turismo ecuatoriano. Ellos ocupan el primer lugar en los comentarios emitidos sobre la ciudad. Dentro del turismo extranjero se destacan los visitantes provenientes de países de América del Sur, entre ellos, Colombia, Perú, Argentina, Brasil, seguido de países de Norte América como Estados Unidos y Canadá y un solo país europeo, como España, y de Oceanía: Australia. Esto refleja una mayor visita de turistas nacionales.

Esta información puede estar sesgada, porque el idioma que se utiliza en las páginas es español; eso puede derivar en que el idioma más utilizado para expresar los comentarios sea éste con un $87.67 \%$, seguido del inglés y portugués (gráfico 7) o evidenciando una gran presencia de turistas con habla hispana y una escasa participación de extranjeros con otro idioma.

\section{Gráfico 7. Totalidad de comentarios según su idioma}

$12,00 \%$

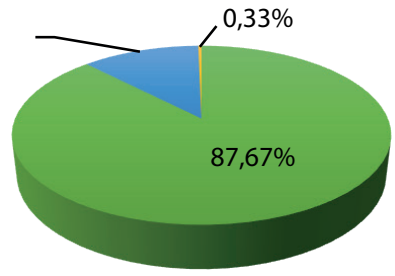

Comentarios en español

Comentarios en Inglés

Comentarios en portugues

Fuente: Elaboración propia.

\section{Discusión y conclusiones}

Los estudios realizados por GIER (2018), muestran que el turismo en la ciudad de Cuenca durante los años 2016-2018, presenta una ligera tendencia al crecimiento de personas procedentes de América Latina y el Caribe con un 1.19\%; y una ligera disminución para Europa (0.5\%), América. del Norte (1.10\%) y 0.4\% para el resto del mundo.

Estos estudios revelan, además, que existe un escaso uso de las páginas webs de las agencias de viajes, tanto nacionales como extranjeras; un 7\% de los turistas obtienen información a través de agencias de viajes, y en mayor proporción por referencias de experiencias anteriores (38.9\%). Mientras que Vidal (2018) afirma que el $74 \%$ de viajeros, hace uso del internet para la planificación de su viaje a través del sitio Google Travel que informa de manera general sobre los sitios turísticos del mundo y solamente el 13\% por agencia de viajes. Existe coincidencia por tanto con los resultados de esta investigación al mostrar (véase Tabla 2) el insuficiente uso de las páginas como medio de información y elección del destino, reflejando una escasa interacción entre los usuarios; éstas deberían ofrecer información como ayuda a la toma de decisiones en la elección del sitio dónde pasar sus vacaciones, en torno a opciones de visita o actividades vinculadas a recursos/atractivos.

En correspondencia con informes anteriores y los hallazgos obtenidos en este trabajo, se confirma que la procedencia de la mayor cantidad de turistas que visitan 
la ciudad, es de países de América Latina y Caribe (Colombia, Perú, Brasil, Argentina, Venezuela, México) con un 78.62\%, seguido de Norte América (EEUU, Canadá) con 17.61\%; en el mismo orden que estableciera GIER (2018), en el que el $35.3 \%$ de fue América Latina seguido por Europa con un 31\% y el americano con un 30.8\%; ello reafirma la viabilidad de utilizar la Netnografía con el empleo de herramientas tecnológicas que derivan en menores costos, en contraposición al levantamiento de información primaria a partir de encuestas.

La observación de las páginas mostró que las páginas web que gestionan los tours operadores, tanto para brindar servicios a turistas nacionales como extranjeras, ofrecen información básica sobre el destino, muy limitada. Es necesario mejorar el contenido de estos sitios y fomentar un espacio de comentarios donde las personas puedan interactuar, expresando sugerencias u opiniones que puedan aportar a la toma decisiones rápida e inmediata por la ciudad como destino, optimizando el tiempo al elegir su viaje; caso contrario, elijan otro lugar como destino.

En este sentido son necesarias las alianzas entre los diferentes actores del turismo, lo cual está expresado en el plan estratégico de turismo de la ciudad (TurisConsulting, 2016, estrategia quinta), donde además se expresa la necesidad de fortalecer a los tours operadores en relación al acceso de mercados y capacidad de negociación; pero lamentablemente esto se queda en declaración de políticas y formulación de estrategias que no se concretan al interior del destino. Una de las actividades que pudiera contribuir a ello es la de incentivar el acceso a sistemas tecnológicos de reservas y compras en línea uniendo esfuerzos a través de la cooperación; en el documento no se evidencian resultados, a pesar de que el mismo contiene un plan de ejecución con responsables y presupuesto.

Desde la óptica de percepción de atractivos, existe también coincidencia con lo publicado por GIER (2018), ya que en ambos se evidencia que las personas aprecian a la ciudad como hermosa, arquitectónica, histórica, el comportamiento de las personas es amable, seguido por los atractivos naturales. Sin embargo se encontró que muy pocas personas eligen a la ciudad por su variedad de iglesias (20 iglesias distribuidas por toda el área urbana), variedad de museos, que es lo que más caracteriza a la ciudad y, por tanto, lo que más se publicita en las páginas o espacios recreativos como Turi, son tours dentro de la ciudad; ello demuestra que el contenido de las páginas deja huecos de información en correspondencia con las preferencias de los turistas, tal como arrojan los estudios de mercado realizados, desestimando el resto de atractivos culturales como otros museos, el entorno natural que rodea a la ciudad su gastronomía o su riqueza cultural en diversas expresiones artísticas.

Los datos de GIER (2018) muestran que los turistas visitan la ciudad de Cuenca motivados por su centro histórico como principal atractivo en un 19\%; esta información coincide con los resultados obtenidos de la investigación (gráfico 5 y 6), reafirmando lo planteado anteriormente. Cabe indicar que en la investigación se definieron otros factores tales como, el clima y seguridad, no obstante, la mayor preferencia con relación al destino es su área arquitectónica que da realce a la ciudad siendo comparada con ciudades europeas.

Con relación a la base de datos extraída, se observa que la mayoría de comentarios están en idioma español (gráfico 7), de manera que los turistas que se expresan 
en otro idioma, no tienen mucha interacción en ellas; esto puede venir dado porque las publicaciones se encuentran solo en este idioma, por tanto, urge publicar los post con otros lenguajes para darle mayor posibilidad de participación a extranjeros de habla no hispana. Esto es importante señalar, ya que las redes sociales se han convertido en medio de conexión entre los negocios y personas; en ellas se comparten ideas, sugerencias, incluso quejas que puedan tener acerca de los productos o servicios que brinda una empresa. Dicha información es relevante ya que arroja necesidades, deseos, sentimientos y aspiraciones de los turistas, y con ello se puede determinar cuáles son sus gustos y preferencias, con el objetivo de mejorar o desarrollar una nueva oferta turística, que satisfaga tanto al turista nacional e internacional, tal como definen autores citados en la parte introductoria.

Aunque no son representativos los datos extraídos con relación a la seguridad, existen señales con respecto a que este puede ser un atractivo de interés especialmente para turistas extranjeros. Estudios realizados por (Prada et al., 2018) apuntan que, la valoración media del viaje, la atención a la población y el aspecto de la ciudad, son valorados de forma positiva y similar en el caso de los "turistas culturales puros" que vistan la ciudad, reafirmando que el contenido de la ciudad está en los niveles de preferencia de los turistas, lo que es significativo para el caso del "turista cultural puro".

Conocer los diferentes atributos de los turistas nacionales y extranjeros es importante para determinar cuál es su comportamiento; la Netnografía es una herramienta válida para este propósito, ya que ayuda a determinar las diferentes conductas e interacciones de los grupos sociales en los diferentes sitios webs con base en la información obtenida de éstas, mediante herramientas tecnológicas que abaratan costos, tal como demuestran las evidencias planteadas en la discusión de los resultados.

La mayoría de los turistas que visitan la ciudad son ecuatorianos, si bien esto tributa a la economía local, sería significativo incrementar el turismo extranjero que contribuye a la entrada de divisas proveniente del turismo foráneo; es ahí donde se deberían enfocar las estrategias para que este segmento continúe moviéndose en una dinámica creciente.

A pesar de que la Fundación Municipal de Turismo de Cuenca estableció cuatro rutas turísticas denominadas Ruta Artesanal, Ruta de los Museos, Ruta de los Molinos y del Pan y la Ruta Rural, con la intención de demostrar que la ciudad de Cuenca tiene otras alternativas turísticas para promover el índice de estancia de los turistas. Estas rutas no han sido promocionadas lo suficiente, dejando de lado la oportunidad que para ello ofrecen las páginas web, aún cuando ha lanzado una aplicación móvil que da a conocer los diversos sitios turísticos de la ciudad entre los que se incluyen los sitios turísticos como iglesias, museos, parques, y otros, con la facilidad de orientarse por GPS.

Todos estos aspectos limitan las oportunidades de mercado, ya que no se aprovecha la diversidad gastronómica, artesanal, cultural, natural que posee la ciudad, una de las causas es que mantiene la forma tradicional de buscar información sobre el comportamiento del consumidor, obviando los avances de la tecnología, especialmente en el ámbito del turismo, donde las personas para elegir o conocer sobre su destino, buscan información mediante las web especialmente en redes sociales que se han convertido en un medio informativo más que social. 
En lo referente a los comentarios obtenidos durante la investigación, se ha observado que la gente visita a la ciudad de Cuenca para diversas actividades recreativas, pero ninguna relacionada al tema de negocios, siendo un punto clave para la ciudad, ya que se fomentaría la participación de varios productores locales con el fin de incrementar la productividad local, en tanto que la Fundación afirma que la ciudad de Cuenca se ve limitada a desarrollarse con una imagen de negocios y convenciones por la falta de conectividad área y capacidad hotelera, descrita en la tercera estrategia del Plan Estratégico (TurisConsulting, 2016).

Otro aspecto importante es que las diferentes opiniones de los turistas acerca de la ciudad son positivas. Muchas personas describen que Cuenca es muy tranquila y destacan el buen trato que reciben de la gente durante su estadía, donde se sienten muy cómodos generando una experiencia única al momento de caminar o tomar un transporte público; dichos comentarios son favorables para la ciudad y el sector turístico; las personas al sentirse más tranquilas generarán buenos comentarios hacia otras personas siendo de gran ayuda e influencia para elegir a Cuenca como su destino, además se incrementará la oportunidad de un retorno y se fomentaría el incremento del turismo.

Tamara (2011) en su investigación ha establecido que la calidez y amabilidad de la gente cuencana a los turistas, es una gran motivación para regresar a la ciudad, los turistas potenciales se ven influenciados por experiencias y/o opiniones de personas que ya han visitado ese destino al momento de tomar decisiones acerca de su viaje (Zeng \& Gerritsen, 2014); las recomendaciones entre familiares y amistades, es la primera fuente de información para la elección del destino, sugiriendo la importancia de brindar buenas experiencias por parte de los prestadores de servicios (Consulting, 2011); uno de los canales donde se expresa esta información, es el virtual, el mismo actúa como influyente a través de comentarios en base a experiencias (Barbery Montoya et al., 2018); estos elementos son claves para que los actores del destino se motiven a desarrollar nuevos productos que permitan aprovechar nuevas oportunidades de mercado.

Vale la pena plantear que la preservación del patrimonio cultural de Cuenca es la génesis para el desarrollo de un destino sostenible, y que coincide con (Prada et al., 2018) cuando afirman que el gasto que implicó la creación de un bien cultural se vuelve baladí frene al costo que demanda su preservación en el tiempo y entre más extensa es la vida del bien cultural, mayor será la inversión que demanda.

\section{Limitaciones}

Las limitaciones del estudio se centran fundamentalmente en la amplitud y profundidad en el levantamiento de los datos con respecto a la selección de la población, dado que en la ciudad existe un bajo nivel de uso de las tecnologías por parte de las empresas de intermediación, lo cual reafirma los resultados de estudios realizados por (García \& Vázquez, 2018).

De otra parte, la obtención de datos personales es limitada debido a que desde el año 2018 en la red social Facebook existe una alta protección de datos (Facebook, 2018), solamente es posible tener acceso al nombre de la persona y ciudad de origen 
restringiendo así, la posibilidad de determinar un perfil completo del turista mediante el uso de la Netnografía, aun cuando (DINARDAP, 2018) afirma que en Ecuador todavía no se han puesto en prácticas, regulaciones al respecto del cumplimiento de la política expresada en la Constitución.

\section{Referencias}

Aucay, E., \& Herrera, P. (2017). Nivel de uso de las redes sociales en el proceso de comunicación en las MIPES de Cuenca. Retos: Revista de Ciencias de la Administración y Economía, 8(14), 81-98. http://dx.doi.org/10.17163/ret.n14.2017.04

Barbery, D. C., Andrade, J., \& Zambrano, M. (2018). Internet y prosumers: impacto en la decisión de compra de servicios hoteleros. Revista Espacios, 39(51). Recuperado de: https://bit. ly/2Z5KVk1 [Fecha de consulta: 22 de junio de 2019].

Castillo, E., Roget, F., \& Rozas, E. (2015). El turismo en Ecuador. nuevas tendencias en el turismo sostenible. Galega de Economía, 24(2), 69-88.

Celdrán, M., Mazón, J., \& Giner, D. (2018). Open Data y turismo. Implicaciones para le gestión turística en ciudades y destinos turísticos inteligentes. Investigaciones Turísticas (15), 49-78. Recuperado de: https://bit.ly/2Ri2aeG

Colordo, D. (2004). Comunidades virtuales. Recuperado de: https://bit.ly/2XOeCJq

Consulting, G. (2011). Plan Estratégico de Desarrollo Turístico. Quito: MINTUR-Fundación Turismo Cuenca.

Dell Innocenti, C. (2012). Las redes sociales digitales como herramientas de marketing. En C. Dell'Innocenti. Mendoza: Universidad de Mendoza.

Desplas, N., \& Mao, M. (2014). Análisis paralelo entre e-turismo y e-gobierno: evolución y tendencias. Investigaciones Turísticas (7), 1-22. Recuperado de: https://bit.ly/2KNI4aU

Dina, R., \& Sabou, G. (2012). Influence of social media in choice of touristic destination. Cactus Tourism Journal, 3(2), 24-30.

DINARDAP (2018). Una Ley de Protección de Datos Personales es una oportunidad para el empresario ecuatoriano. Recuperado de: https://bit.ly/2vxpnje

Facebook (2018). Compromiso de Facebook con la protección de datos y la privacidad en cumplimiento del GDPR. Recuperado de: https://bit.ly/2EGyMvB

Fisher, T. (2009). ROI in social media: A look at the arguments. Journal of Database Marketing \& Customer Strategy Management, 16(3), 189-195. https://doi.org/10.1057/dbm.2009.16

García, F., \& Vázquez, J. (2018). MIPYMES distribuidoras turísticas: recomendaciones seo a partir del análisis de palabras claves. Cuenca: Grupo Investigación Gestión de las MIPYMES-GIGMP.

Gebera, W. (2008). La netnografía: un método de investigación. Barcelona: EDUCAR. Recuperado de: https://bit.ly/2JAO439 (2018- 01-12). [Fecha de consulta: 12 de enero de 2018].

GIER (2018). Anuario Estadístico Estudio de Demanda y Oferta Turística en la ciudad de Cuenca. Cuenca: Universidad de Cuenca. Recuperado de: https://bit.ly/2KgMp7f

Gutiérrez, G., Sánchez, M., \& Galiano, A. (2018). Redes sociales como medio de promoción turística en los países iberoamericanos. Retos Revista de Ciencias de la Administración y Economía, 15(8), 135-150. https://doi.org/10.17163/ret.n15.2018.09

INEC (2017). Tecnologías de la información y comunicación. Recuperado de: https://bit.ly/2PS8kRz

Kang, M., \& Schuett, M. (2013). Determinants of sharing travel experiences in social media. Journal of Travel \& Tourism Marketing, 30(1-2), 93-107. https://doi.org/10.1080/1054840 8.2013.751237

Marín, P., \& Torres, Á. (2017). Gamificación en aplicaciones móviles para servicios bancarios de España. Retos: Revista de Ciencias de la Administración y Economía, VII(13). https://doi. org/10.17163/ret.n13.2017.02 
MINTUR (17 de agosto de 2017). Ecuador se promocionará inteligentemente a nivel mundial, con alianza estratégica con Amadeus. Recuperado de: https://bit.ly/2x8pqlX

Montserrat, G. (2014). Impacto de las TIC en el turismo. Valladolid.

Morales, A. P. (2016). Uso de redes sociales como estrategia de promoción de marketing. Cuenca: Universidad de Cuenca.

Prada, J., Armijos, D., Crespo, A., \& Torres, L. (2018). El turista cultural: tipologías y análisis de las valoraciones del destino a partir del caso de estudio de Cuenca-Ecuador. Pasos: Revista de Turismo y Patrimonio Cultural, 16(1), 55-72. https://doi.org/10.25145/j.pasos.2018.16.004

Quezada, P. A., Suasnavas, M. G., Chango-Canaveral, P. M., Gonzaga-Vallejo, C., Enciso, L., \& Calderón-Cordova, C. A. (2018). Used of Social Networks and web application to design and promote the ecotourism route in the Southern Amazon of Ecuador. 13th Iberian Conference on Information Systems and Technologies, CISTI, june, 27, pp. 1-7. doi:10.23919/CISTI.2018.8399399

Quijano, C., Arellano, A., \& Naranjo, K. (2017). (Dis)connection during a tourism trip: The use of smartphones by independent travelers. Guayaquil: ESPOL.

Romeo, A., Abad, J., Forgas, S., \& Huertas, R. (2014). La netnografía como herramienta de investigación en contextos on-line. Innovar Journal, 24(52), 89-102.

Sakulsureeyadej, A. (2011). Cómo la tecnología está cambiando el turismo mundial. En OMT, AM Reports: Tecnología y Turismo (Vol. 1). Recuperado de: https://bit.ly/2P2i5uZ

Saura, J., Sánchez, P., \& Reyes, A. (2017). Marketing a través de aplicaciones móviles de turismo (m-tourism). Un estudio exploratorio. Madrid.

SENPLADES (2012). Transformación de la Matriz Productiva: Revolución productiva a través del conocimiento y el talento humano (1 ed.). Quito.

Sotiriadis, M., \& Van Zyl, C. (2013). Electronic word-of-mouth and online reviews in tourism services: the use of twitter by tourists. Electronic Commerce Research, 13, 103-124. https:// doi.org/10.1007/s10660-013-9108-1

Tafur, G., Vélez, C., Machado, O., Zumba, M., \& Jácome, J. (2018). Desarrollo tecnológico del sector turístico en la ciudad de Guayaquil (Ecuador). Revista Espacios, 39(44), 3. Recuperado de: https://bit.ly/2XSnJ7n (2019-06-22).

Tamara, F. (2011). Guía para la creación de emprendimientos culturales. Cuenca: Universidad de Cuenca. Recuperado de: https://bit.ly/2sErF1p

TurisConsulting (2016). Plan Estratégico de Desarrollo Turístico y Mercadeo del destino Cuenca y su área de Influencia 2016-2021 (Vol. 1). Cuenca.

Vargas, G. (2011). Método Netnográfico. En G. M. Vargas, La investigación de mercados online y la Netnografía (pp. 42-43). Santiago de Chile: Universidad de Chile.

Vázquez, J. P. (2018). Aportes al comercio turístico a través de websites: Mejoramiento para la evaluación de calidad. Un espacio para la ciencia, 1(1), 145-168. Recuperado de: https:// bit.ly/2KlZrjx

Vidal, B. (2018). Turismo y tecnología: cómo la tecnología revoluciona el sector turístico. Recuperado de: https://bit.ly/2M8ZIDU

Zeng, B., \& Gerritsen, R. (2014). What do we know about social media in tourism? A review. Tourism Managment Perspectives, 10, 27-36. https://doi.org/10.1016/j.tmp.2014.01.001 